\title{
Efectividad de una intervención cognitiva en funciones ejecutivas para mejorar flexibilidad mental en niños con trastorno por déficit de atención e hiperactividad
}

\author{
Effectiveness of a cognitive intervention in executive functions to improve \\ mental flexibility in children with attention deficit disorder and hyperactivity
}

Ángela Magnolia Ríos-Gallardo', Alfredis González Hernández', Diana Carolina Toledo Nieto', María Alejandra Bermeo Losada', Jasmín Bonilla-Santos², Lísimaco Vallejo Cuéllar ${ }^{3 *}$

\begin{abstract}
Resumen
El trastorno por Déficit de Atención e Hiperactividad (TDAH) es un síndrome conductual que se presenta en la infancia, sobre todo en la población masculina, y se caracteriza por hiperactividad, impulsividad, y en ocasiones, está acompañado de inatención y un funcionamiento inapropiado de acuerdo a las exigencias del contexto. Es la afección comportamental más frecuente en la población escolar, tiene su inicio en la infancia y representa un problema de alta complejidad dado que incide en las actividades cotidianas del niño que lo padece. Es bastante probable que dicha afectación persista a lo largo de la vida, si no es tratada a tiempo. Se estima que 5,1 millones de niños poseen diagnóstico de TDAH en el mundo. La prevalencia global de este trastorno oscila entre el $3 \%$ y $7 \%$. Objetivo: Determinar la eficacia de un programa de estimulación cognitiva en las funciones ejecutivas para mejorar la flexibilidad mental en niños con TDAH. Metodología: Tipo de estudio cuantitativo, cuasiexperimental, con grupo control, pretestentrenamiento-postest. Los participantes del estudio fueron 51 niños escolarizados, 34 diagnosticados con TDAH (grupo experimental) y 17 niños que no presentan ningún síntoma que sugiera alteraciones comportamentales (grupo control). Resultados: El grupo experimental mostró beneficiarse del entrenamiento y esto evidencia la ganancia significativa con respecto al grupo control en pruebas que miden el sistema ejecutivo.
\end{abstract}

Palabras clave: Trastorno de hiperactividad y déficit de atención, sistema ejecutivo central, control inhibitorio.

\begin{abstract}
The Attention Deficit Hyperactivity Disorder (ADHD) is a behavioral syndrome that occurs in childhood, especially in the male population, is characterized by hyperactivity, impulsivity, and sometimes, accompanied by inattention and inappropriate operation according to the demands of the context. This behavior is more frequent in the school population, that has its beginning in childhood and it affects the activities of the child who suffers from it. It is quite probable that this affectation persists to throughout life, if not treated on time. It is estimated that 5.1 million children have a diagnosis of ADHD in the
\end{abstract}

1 Grupo de Investigación Dneuropsy, Universidad Surcolombiana.

2 Grupo de Investigación Dneuropsy, Universidad Cooperativa de Colombia-Sede Neiva.

3 Grupos de investigación Inefc, Barcelona.

* Correspondencia: Ph.D. Lisímaco Vallejo Cuéllar. Correo electrónico: lisvac@gmail.com

Recibido: 30/09/2014 - Revisado: 06/07/2015 - Aceptado: 16/03/2016 
Efectividad de una intervención cognitiva en funciones ejecutivas para mejorar flexibilidad mental en niños con trastorno por déficit de atención e hiperactividad
R.F.S Revista Facultad de Salud

Enero - Junio de 2016;8(1): 16-22

world. The global prevalence of this disorder ranges from $3 \%$ to $7 \%$. Objective: To determine the effectiveness of a cognitive stimulation program in executive functions to improve mental flexibility in children with ADHD. Methodology: Type of quantitative study, quasi-experimental, with control group, pretest-training-postest. Materials and methods: The participants of the study were 51 children school age, 34 diagnosed with ADHD (experimental group) and 17 children who do not present any symptoms that suggests behavioral alterations (control group) Results: The experimental group showed to benefit from training and this evidence the significant gain with respect to the control group in tests that measure the executive system.

Key words: Attention deficit disorder with hyperactivity, central executive system, inhibitory control.

\section{Introducción}

Los niños que son diagnosticados con TDAH pueden presentar desajustes importantes que inciden en el desempeño normal de sus actividades cotidianas, debido a las falencias de tipo atencional que generalmente están ligadas a hiperactividad e impulsividad. El DSM-IV define el TDAH como un trastorno de inicio en la infancia, caracterizado por la presencia de un patrón persistente de desatención o hiperactividad-impulsividad ${ }^{(1)}$, el cual es más frecuente y grave que el observado en personas con similar nivel de desarrollo. Dichos síntomas deben haberse instaurado antes de los siete años de edad. Al analizar esta población en su funcionamiento cognitivo, estos muestran un perfil caracterizado principalmente por un déficit en las funciones ejecutivas, como consecuencia de un compromiso del córtex prefrontal y conexiones subcorticales ${ }^{(2)}$. Las funciones neuropsicológicas involucradas en estas áreas cerebrales analizadas con técnicas de neuroimagen han mostrado la existencia de una disfunción en una red frontoestriatal en este trastorno, lo que podría estar mediando las alteraciones observadas en procesos ejecutivos como, por ejemplo, la inhibición de respuesta ${ }^{(3)}$. Otras técnicas de imagenología funcional apoyan la hipótesis de alteraciones en el funcionamiento de los circuitos neurales entre los lóbulos frontales, el cuerpo estriado y el cerebelo en el mecanismo fisiopatológico del $\mathrm{TDAH}^{(4)}$.

Al comparar los hallazgos anatomofuncionales del TDAH es necesario analizar la relación que estos tienen con el funcionamiento cognitivo. Diversas investigaciones consideran importante estudiar el funcionamiento ejecutivo central dada la implicación de áreas cerebrales relacionadas con dicha función. El sistema ejecutivo central supervisa y ordena la información proveniente de los dos "sistemas buffers" y sirve para seleccionar una parte de la información a fin de realizar las pruebas cognitivas más exigentes ${ }^{(5)}$.

Asimismo, el funcionamiento de los lóbulos frontales se asocia con niveles más elevados de la función cortical, con inclusión de aquellos inherentes a la actividad intelectual, tales como una conducta orientada a una meta y la planificación conductual autodirigida ${ }^{(5,6)}$, esto sugiere que los niños que presentan TDAH pueden llegar a presentar alteraciones de tipo atención sostenida y discriminación atencional, de modo que son susceptibles a perder el foco por interferencia ambiental irrelevante. Es por esto que desde los años noventa, Rothenberger y Etchepareborda plantearon que una forma precisa de llegar al diagnóstico de TDAH proviene de la documentación de las funciones cerebrales frontales que incluyen: las funciones atencionales (estado de alerta, atención sostenida y la discriminación atencional), el control de impulsos, el control de interferencia y la flexibilidad cognitiva ${ }^{7}$, ${ }^{8)}$. El objetivo del presente estudio fue determinar la eficacia de un programa de intervención cognitiva en las funciones ejecutivas para mejorar la flexibilidad mental en niños con TDAH.

\section{Materiales y método}

La investigación adoptó un diseño cuasiexperimental pretestpostest con grupo control. Se seleccionaron 51 niños escolarizados entre 6 y 15 años que asistían a dos escuelas públicas de la ciudad de Neiva. El nivel de escolaridad de los participantes osciló entre segundo de primaria a séptimo de secundaria. Se conformó un grupo experimental con diagnóstico de TDAH (grupo experimental $n=34,28$ hombres y 6 mujeres con una de edad $=9,62 \pm 2,1$ años) y un grupo control sin características clínicas (grupo control $\mathrm{n}=17,4$ hombres y 13 mujeres con una edad $=10,6 \pm 2,4$ años). (Tabla 1). El grupo experimental se seleccionó de acuerdo con los criterios clínicos para diagnosticar el TDAH (criterios diagnósticos DSMIV Diagnostic and Statistical Manual of Mental Disorders). El grupo control no presentó antecedentes a nivel clínico del TDAH, según reporte de familias y docentes.

\section{Instrumentos}

Para analizar el sistema ejecutivo central se aplicaron tres pruebas. El Test de Clasificación de Tarjetas de Wisconsin $(\mathrm{WCST})^{(9)}$, una prueba que permite obtener la medida de la función ejecutiva que requiere estrategias de planificación, indagaciones organizadas y utilización de la retroalimentación ambiental para cambiar esquemas. Dada su posible sensibilidad a los efectos de las lesiones en el lóbulo frontal, se menciona frecuentemente como una medida del funcionamiento frontal o prefrontal. Otro instrumento aplicado fue el Test de Colores y Palabras (STROOP) ${ }^{(10)}$, el cual mide la organización del aprendizaje. Con esta prueba se busca in- 
R.F.S Revista Facultad de Salud

Enero - Junio de 2016;8(1): 16-22
Efectividad de una intervención cognitiva en funciones ejecutivas para mejorar flexibilidad mental en niños con trastorno por déficit de atención e hiperactividad

Tabla 1. Características demográficas de la población estudiada

\begin{tabular}{|c|c|c|c|c|c|c|c|}
\hline & \multirow{2}{*}{ Variables } & \multicolumn{3}{|c|}{ Grupo Experimental } & \multicolumn{3}{|c|}{ Grupo Control } \\
\hline & & Media & DS & Rango & Media & DS & Rango \\
\hline \multirow{2}{*}{\multicolumn{2}{|c|}{ Edad }} & 9,62 & 2,1 & $6-15$. & 10,06 & 2,04 & $6-15$ \\
\hline & & Frecuencia & & centaje & Frecuencia & & rcentaje \\
\hline \multirow[t]{5}{*}{ Género } & Masculino & 28 & & 54,9 & 4 & & 7,8 \\
\hline & Femenino & 6 & & 11,7 & 13 & & 25,4 \\
\hline & Segundo de primaria & 6 & & 11,8 & 1 & & 1,96 \\
\hline & Tercero de primaria & 4 & & 7,84 & 2 & & 3,92 \\
\hline & Cuarto de primaria & 13 & & 25,5 & 5 & & 9,8 \\
\hline \multirow[t]{4}{*}{ Escolaridad } & Quinto de primaria & 4 & & 7,8 & 4 & & 7,8 \\
\hline & Sexto de secundaria & 3 & & 5,88 & 1 & & 1,96 \\
\hline & Séptimo Secundaria & 4 & & 7,8 & 4 & & 7,8 \\
\hline & Total & 34 & & 66,6 & 17 & & 33,24 \\
\hline
\end{tabular}

Fuente: Elaboración de los autores.

hibir la tendencia automática y responder de manera controlada mediante la solución de estímulos en conflicto $^{(11)}$. Finalmente, se utilizó una prueba denominada Tarea Dual (método Go/no Go), que permite evaluar velocidad de procesamiento, efecto atencional, amplitud de memoria, diferencia la velocidad o capacidad de procesamiento del control o memoria de trabajo.

\section{Procedimiento}

El estudio se divide en tres etapas. Inicialmente se evaluaron los procesos cognitivos del grupo experimental y el grupo control (pretest). Luego se aplicó al grupo experimental el programa de intervención, y una vez finalizadas las actividades de intervención, se aplicaron nuevamente los test a los dos grupos de estudio (postest).

El programa de intervención cognitiva y comportamental de las funciones ejecutivas, que se diseñó luego de la la evaluación inicial, estuvo orientado a fortalecer la flexibilidad cognitiva de los niños con TDAH. El programa fue aplicado al grupo experimental en 15 sesiones de trabajo con frecuencia de una sesión semanal y duración de dos horas cada una. La aplicación del programa de intervención cognitiva se adaptó a las características individuales de los participantes, con el fin de que resultara más atractivo y motivante para cada uno de ellos. Además, se basó en actividades de juego y tareas que realizaban los niños cotidianamente, y se tomó como principio la estimulación de diferentes canales sensoriales que permitieran un mayor grado de atención y desarrollo de la función ejecutiva (flexibilidad cognitiva).
El protocolo de investigación y el consentimiento informado fueron aprobados por el Comité de Ética en Investigación de la Facultad de Salud de la Universidad Surcolombiana. En la elaboración de este proyecto investigativo se retomaron los principios éticos expuestos en la Declaración de Helsinki, que a su vez son consistentes con la Buena Práctica Clínica, además de lo estipulado en la Resolución 8430 de 1993 y el Código Deontológico y Bioético y otras disposiciones de la profesión de Psicología (Ley 1090 de 2006).

\section{Resultados}

El programa de intervención de estimulación cognitiva y comportamental de las funciones ejecutivas fue eficaz para mejorar la flexibilidad mental, la atención, planeación, control inhibitorio y la memoria para las siguientes variables: Test Wisconsin, la prueba digital Tarea Dual 2 Go/no Go y la prueba de STROOP para la categoría palabra. Para el Test Wisconsin se encontraron diferencias significativas en: aciertos, errores, categorías, perseveración color, perseveración forma, total perseveraciones y errores de otras categorías (Tabla 2).

Así mismo, para la prueba digital Tarea Dual 2 Go/no Go, se encontraron diferencias significativas en la variable TR erradas (Tabla 3); y finalmente en la prueba STROOP categoría palabra se observó una diferencia en la variable número de errores congruentes (Tabla 4).

Estas variables obtuvieron diferencias significativas entre el pretest y postest para el grupo experimental, con un nivel de probabilidad de $p=0.05$. Sin embargo, las siguientes variables no mostraron diferencias significativas, como las 
Tabla 2. Comparación intergrupo puntuaciones pre-post Test Wisconsin

\begin{tabular}{|c|c|c|c|c|c|c|c|}
\hline \multicolumn{5}{|c|}{ Grupo Experimental $\mathrm{N}=32$} & \multicolumn{3}{|c|}{ Grupo Control N=17 } \\
\hline \multicolumn{2}{|l|}{ Test Wisconsin } & Media & Std. Dv. & $\mathbf{p}$ & Media & Std. Dv. & $\mathbf{p}$ \\
\hline \multirow{2}{*}{ Aciertos } & pretest & 22,21 & 6,49 & \multirow{2}{*}{0,000} & 19,41 & 5,84 & \multirow{2}{*}{0,29} \\
\hline & postest & 31,03 & 5,25 & & 20,20 & 6,31 & \\
\hline \multirow{2}{*}{ Errores } & pretest & 25,56 & 6,57 & \multirow{2}{*}{0,000} & 28,35 & 5,71 & \multirow{2}{*}{0,90} \\
\hline & postest & 15,87 & 4,32 & & 29,76 & 6,63 & \\
\hline \multirow{2}{*}{ Categorías } & pretest & 2,03 & 1,30 & \multirow{2}{*}{0,000} & 1,82 & 0,88 & \multirow{2}{*}{0,80} \\
\hline & postest & 3,87 & 1,38 & & 1,95 & 1,16 & \\
\hline \multirow{2}{*}{ Perseveración color } & pretest & 3,84 & 2,94 & \multirow{2}{*}{0,008} & 4,94 & 3,61 & \multirow{2}{*}{0,69} \\
\hline & postest & 2,25 & 1,96 & & 5,41 & 6,30 & \\
\hline \multirow{2}{*}{ Perseveración forma } & pretest & 3,31 & 5,93 & \multirow{2}{*}{0,041} & 2,52 & 2,91 & \multirow{2}{*}{0,17} \\
\hline & postest & 1,31 & 1,37 & & 1,58 & 1,46 & \\
\hline \multirow{2}{*}{ Total perseveraciones } & pretest & 9,34 & 5,69 & \multirow{2}{*}{0,000} & 10,35 & 3,29 & \multirow{2}{*}{0,83} \\
\hline & postest & 4,96 & 2,84 & & 10 & 6,26 & \\
\hline \multirow[b]{2}{*}{ Errores de otras categorías } & pretest & 1,71 & 2,79 & \multirow[b]{2}{*}{0,013} & 2,94 & 3,50 & \multirow[b]{2}{*}{0,19} \\
\hline & postest & 0,31 & 0,85 & & 2,05 & 2,81 & \\
\hline
\end{tabular}

Fuente: Elaboración de los autores.

Tabla 3. Comparación intergrupo puntuaciones pre-post Tarea Dual 2 Go/no Go.

\begin{tabular}{|c|c|c|c|c|c|c|c|}
\hline \multirow{2}{*}{$\begin{array}{c}\text { Prueba digital } \\
\text { Tarea Dual } 2 \text { Go/no Go } \\
\end{array}$} & & \multicolumn{3}{|c|}{ Grupo Experimental $\mathbf{N}=32$} & \multicolumn{3}{|c|}{ Grupo Control N=17 } \\
\hline & & Media & Std. Dv. & $\mathbf{p}$ & Media & Std. Dv. & $\mathbf{p}$ \\
\hline \multirow{2}{*}{ Tiempo R Erradas } & pretest & 281,93 & 352,47 & \multirow{2}{*}{0,000} & 323,48 & 329,33 & \multirow{2}{*}{0,101} \\
\hline & postest & 72,74 & 190,32 & & 169,39 & 248,36 & \\
\hline \multirow[b]{2}{*}{ Ensayos correctos } & pretest & 23,12 & 8,31 & \multirow{2}{*}{0,077} & 23,58 & 7,14 & \multirow{2}{*}{0,258} \\
\hline & postest & 24,96 & 5,75 & & 20,52 & 7,14 & \\
\hline \multirow[b]{2}{*}{ Error posición } & pretest & 0,62 & 1,15 & \multirow{2}{*}{0,271} & 3,82 & 7,96 & \multirow{2}{*}{0,292} \\
\hline & postest & 0,34 & 0,97 & & 1,41 & 3,58 & \\
\hline \multirow[b]{2}{*}{ Error freno } & pretest & 1,25 & 2,66 & \multirow{2}{*}{0,832} & 3,88 & 7,03 & \multirow{2}{*}{0,214} \\
\hline & postest & 1,40 & 3,08 & & 1,47 & 1,97 & \\
\hline \multirow[b]{2}{*}{ Omisión } & pretest & 1,40 & 3,29 & \multirow{2}{*}{0,659} & 2,70 & 4,99 & \multirow{2}{*}{0,136} \\
\hline & postest & 1,25 & 2,32 & & 5,47 & 4,11 & \\
\hline \multirow[b]{2}{*}{ Tiempo de respuesta correcta } & pretest & 592,84 & 174,39 & \multirow{2}{*}{0,716} & 599,51 & 68,83 & \multirow{2}{*}{0,400} \\
\hline & postest & 601,32 & 134,84 & & 909,38 & 1493,51 & \\
\hline
\end{tabular}

Fuente: Elaboración de los autores. 
R.F.S Revista Facultad de Salud Enero - Junio de 2016;8(1): 16-22
Efectividad de una intervención cognitiva en funciones ejecutivas para mejorar flexibilidad mental en niños con trastorno por déficit de atención e hiperactividad

Tabla 4. Comparación intergrupo puntuaciones pre-post Prueba STROOP.

\begin{tabular}{|c|c|c|c|c|c|c|c|}
\hline \multirow[b]{2}{*}{$\begin{array}{c}\text { STROOP } \\
\text { Categoría Palabra }\end{array}$} & & \multicolumn{3}{|c|}{ Grupo Experimental $\mathbf{N}=32$} & \multicolumn{3}{|c|}{ Grupo Control N=17 } \\
\hline & & Media & Std. Dv. & $\mathbf{p}$ & Media & Std. Dv. & $\mathbf{p}$ \\
\hline \multirow{2}{*}{$\begin{array}{l}\text { No Errores } \\
\text { Congruentes }\end{array}$} & pretest & 0,15 & 0,44 & \multirow{2}{*}{0,044} & 0,88 & 2,02 & \multirow[b]{2}{*}{0,22} \\
\hline & postest & 0,03 & 0,17 & & 0,23 & 0,75 & \\
\hline \multirow[b]{2}{*}{$\begin{array}{l}\text { Tiempo respuesta } \\
\text { palabra correcta }\end{array}$} & pretest & 1695,08 & 647,89 & \multirow{2}{*}{0,482} & 1596,00 & 616,05 & \multirow{2}{*}{0,267} \\
\hline & postest & 1546,97 & 790,90 & & 2286,85 & 2417,92 & \\
\hline \multirow{2}{*}{$\begin{array}{l}\text { Tiempo respuesta } \\
\text { palabra congruente }\end{array}$} & pretest & 1638,23 & 918,29 & \multirow{2}{*}{0,332} & 1477,436 & 643,544 & \multirow{2}{*}{0,952} \\
\hline & postest & 1428,70 & 593,60 & & 1486,882 & 526,588 & \\
\hline \multirow{2}{*}{$\begin{array}{l}\text { Palabra error } \\
\text { congruente }\end{array}$} & pretest & 14,21 & 2,76 & \multirow{2}{*}{0,134} & 14,118 & 2,027 & \multirow{2}{*}{0,826} \\
\hline & postest & 14,96 & 0,17 & & 13,882 & 3,655 & \\
\hline \multirow{2}{*}{$\begin{array}{l}\text { Palabra tiempo error } \\
\text { congruente }\end{array}$} & pretest & 143,17 & 460,87 & \multirow{2}{*}{0,086} & 272,906 & 1018,110 & \multirow{2}{*}{0,470} \\
\hline & postest & 10,62 & 60,10 & & 73,063 & 292,250 & \\
\hline \multirow{2}{*}{$\begin{array}{l}\text { Palabra tiempo } \\
\text { respuesta incongruente }\end{array}$} & pretest & 1755,08 & 640,06 & \multirow{2}{*}{0,885} & 1751,584 & 588,785 & \multirow{2}{*}{0,804} \\
\hline & postest & 1788,80 & 966,53 & & 1689,198 & 706,656 & \\
\hline \multirow{2}{*}{$\begin{array}{l}\text { Palabra respuesta } \\
\text { correcta incongruente }\end{array}$} & pretest & 12,18 & 4,24 & \multirow{2}{*}{0,232} & 11,059 & 5,190 & \multirow{2}{*}{0,900} \\
\hline & postest & 13,12 & 2,12 & & 11,235 & 4,338 & \\
\hline \multirow{2}{*}{$\begin{array}{l}\text { Palabra error } \\
\text { incongruente }\end{array}$} & pretest & 2,34 & 3,64 & \multirow{2}{*}{0,474} & 4,118 & 5,023 & \multirow{2}{*}{0,264} \\
\hline & postest & 1,87 & 2,12 & & 2,882 & 3,314 & \\
\hline \multirow{2}{*}{$\begin{array}{l}\text { Palabra tiempo error } \\
\text { incongruente }\end{array}$} & pretest & 1041,06 & 1139,44 & 0762 & 1461,264 & 1031,400 & 0.308 \\
\hline & postest & 1108,98 & 914,27 & 0,102 & 1128,802 & 826,230 & 0,308 \\
\hline Color tiempo respuesta & pretest & 1649,56 & 719,80 & 0383 & 1484,960 & 598,140 & 0.541 \\
\hline correcta & postest & 1537,72 & 781,70 & 0,383 & 1358,974 & 754,489 & 0,341 \\
\hline Color tiempo respuesta & pretest & 1466,64 & 605,10 & 0521 & 1297,346 & 560,535 & 0830 \\
\hline correcta & postest & 1401,74 & 737,54 & $0,5<1$ & 1254,612 & 751,776 & 0,000 \\
\hline Color respuesta & pretest & 14,00 & 3,68 & 0205 & 14,882 & 0,332 & 0150 \\
\hline correcta congruente & postest & 14,46 & 2,65 & 0,299 & 13,059 & 4,930 & 0,150 \\
\hline Color error conoruente & pretest & 0,06 & 0,24 & 1 & 0,118 & 0,332 & 0579 \\
\hline coror error congruente & postest & 0,06 & 0,24 & 1 & 0,176 & 0,393 & $0,0 / 9$ \\
\hline Color tiempo error & pretest & 85,12 & 338,79 & 0426 & 305,794 & 995,063 & 0969 \\
\hline congruente & postest & 30,06 & 170,05 & 0,720 & 316,941 & 594,380 & , \\
\hline Color tiempo respuesta & pretest & 2039,02 & 1490,94 & 0556 & 1913,196 & 1692,832 & 0301 \\
\hline incongruente & postest & 1849,24 & 1150,50 & 0,5J0 & 1475,871 & 801,734 & 0,501 \\
\hline Color respuesta & pretest & 11,71 & 4,01 & 0416 & 11,118 & 5,207 & 0949 \\
\hline correcta incongruente & postest & 12,46 & 4,11 & 0,410 & 11,235 & 5,250 & $0,4+9$ \\
\hline
\end{tabular}


Continuación Tabla 4.

Grupo Experimental N= 32

Grupo Control N=17

\begin{tabular}{lccccccc}
\hline $\begin{array}{c}\text { STROOP } \\
\text { Categoría Palabra }\end{array}$ & & Media & Std. Dv. & p & Media & Std. Dv. & p \\
\hline $\begin{array}{l}\text { Color error } \\
\text { incongruente }\end{array}$ & pretest & 2,34 & 2,64 & 0,694 & 3,882 & 5,207 & 0,207 \\
\hline Color tiempo error & postest & 2,03 & 3,45 & & 2,000 & 3,202 & \\
incongruente & pretest & 1558,35 & 830,31 & 0,895 & 1362,491 & 912,535 & 0,961 \\
\hline
\end{tabular}

Fuente: Elaboración de los autores.

del Test de Wisconsin Perseveración número, Perseveración otras, La prueba Digital en la subescala de la Tarea Dual 1, CPT (Ensayos correctos, ensayos errados, omisiones, falsas alarmas, TR correctas y TR erradas). Para la Tarea Dual 2 Go/ no Go (ensayos correctos, errores de posición, errores freno, omisiones y TR correctas). Para la prueba digital Tarea Dual 3 Tiempos (Dual Tarea 1 y Dual Tarea 2). Como también para la Prueba Digital Tarea Dual 3, Tarea 1-2 (ensayos correctos SPT, ensayos correctos Go/no Go, ensayos errados, errores posición, errores frenos y omisiones). Para la Prueba de STROOP categoría palabra (TR correctas total, TR congruentes, número de respuestas correctas congruentes, TR errores congruentes, TR incongruentes, número de respuestas correctas incongruentes, número de errores incongruentes, TR errores incongruentes). Para la Prueba Stroop, categoría color, tampoco se encontraron diferencias significativas en todas subescalas. Para el grupo control, los datos se mantuvieron estables o sin diferencias estadísticamente significativas.

\section{Discusión}

El objetivo del estudio fue determinar la eficacia de un programa de intervención cognitiva en funciones ejecutivas para mejorar la flexibilidad mental. Los resultados de la comparación pretest-postest evidenciaron que el programa de intervención cognitiva y comportamental de las funciones ejecutivas fue eficaz para mejorar los resultados de la pruebas Test Wisconsin, la prueba digital Tarea Dual 2 Go/no Go y la prueba de STROOP para la categoría palabra, en niños escolarizados con diagnóstico de TDAH.

El estudio reveló que los niños con TDAH presentaban dificultades en el funcionamiento ejecutivo, procesos atencionales y flexibilidad mental. Previamente, Fei Li et al., utilizando técnicas de neuroimagen para explorar las alteraciones de funciones neurales a nivel de red y regional en una muestra de niños con TDAH, encontraron alteraciones en la corteza orbitofrontal, que participa principalmente en el procesamiento cognitivo de la planificación estratégica, y en el globo pálido, estructura implicada en el control inhibitorio ${ }^{(12)}$.
Es decir que las anormalidades estructurales y funcionales en estas regiones cerebrales pueden causar la falta de atención y la hiperactividad de los pacientes con TDAH. Adicionalmente, se hallaron anormalidades en las conexiones entre las redes del cerebro en estado de reposo asociadas con la disfunción ejecutiva. Estas anomalías indican alteraciones cerebrales más extensas en el TDAH de lo que se había demostrado.

Por su parte, Delgado y Etchepareborda en su estudio sobre los trastornos de las funciones ejecutivas, su diagnóstico y tratamiento, identificaron el síndrome prefrontal afectado $y$ especificaron el funcionamiento ejecutivo como un elemento fundamental para el establecimiento de protocolos de intervención neuropsicológica, el cual debe estar a su vez soportado desde herramientas válidas y fiables que permitan abordar los síndromes prefrontales desde múltiples canales sensoriales y en todos sus sistemas de procesamiento de la información $^{(13)}$.

El programa de intervención cognitiva y comportamental de las funciones ejecutivas que se implementó en este estudio, se orientó a fortalecer el funcionamiento ejecutivo de los niños, específicamente las habilidades de flexibilidad mental mediante actividades que requerían la planificación, verificación, y resolución de problemas. Además, el programa siguió los lineamientos de Abad-Mas, Ruiz-Andrés, Moreno-Madrid, Herrero y Suay $2013^{(14)}$, los cuales recomiendan que la intervención psicopedagógica en niños con TDAH debe contemplar la individualización del tratamiento dentro de una metodología multidisciplinar, teniendo en cuenta todos los contextos en los que se desarrolla el niño, su rendimiento cognitivo y las intervenciones apropiadas en cada caso.

Los resultados evidenciaron diferencias significativas $(\mathrm{P}<.005)$ a favor del grupo experimental en la ejecución de tareas de planificación, control inhibitorio, flexibilidad cognitiva y memoria de trabajo. Estas diferencias se observaron en el contexto cotidiano de los niños mediante la mejoría en la ejecución de actividades que exigen demandas atencionales. Sin embargo, los resultados obtenidos se limitan a los niños con TDAH participantes del estudio; los efectos alcanzados no son generalizables. En futuras 
R.F.S Revista Facultad de Salud

Enero - Junio de 2016;8(1): 16-22
Efectividad de una intervención cognitiva en funciones ejecutivas para mejorar flexibilidad mental en niños con trastorno por déficit de atención e hiperactividad investigaciones, se sugiere una muestra mayor que permita la generalización de los resultados.

\section{Conclusión}

El programa de intervención de estimulación cognitiva y comportamental de las funciones ejecutivas fue efectivo para mejorar la flexibilidad cognitiva, la atención, planeación, control inhibitorio y la memoria de trabajo, resultados que se evidenciaron en las diferencias significativas en tareas del Test Wisconsin, la prueba digital Tarea Dual 2 Go/no Go y la prueba de STROOP. Además, debido a los cambios en funcionamiento ejecutivo, los niños desarrollaran mayores períodos atencionales necesarios para realizar actividades cognitivas complejas como las acciones escolares (lectura, cálculo y escritura), y la apropiación de estrategias de planificación en las tareas de la vida cotidiana.

\section{Referencias}

1. VISSER SN, DANIELSON ML, BITSKO RH, HOLBROOK JR, KOGAN MD, GHANDOUR RM, ET AL. Trends in the parent-report of health care provider-diagnosed and medicated attention-deficit/hyperactivity disorder: United States, 2003-2011. Journal of the American Academy of Child \& Adolescent Psychiatry. 2014;53(1):34-46.

2. TRUJILLO-ORREGO N, PINEDA DA, URIBE LH. Validez del diagnóstico de trastorno por déficit de atención/ hiperactividad: de lo fenomenológico a lo neurobiológico (I). Rev Neurol. 2012;54:289-302.

3. FILIPPETTI V, MÍAS C. Neuropsicología del trastorno por déficit de atención/hiperactividad: subtipos predominio déficit de atención y predominio hiperactivo-impulsivo. Revista Argentina de Neuropsicología. 2009;13:14-28.

4. LAWRENCE V, HOUGHTON S, DOUGLAS G, DURKIN K, WHITING K, TANNOCK R. Executive function and ADHD: A comparison of children's performance during neuropsychological testing and real-world activities. Journal of attention disorders. 2004;7(3):137-49.

5. BADDELEY AD, HITCH G. Working memory. The psychology of learning and motivation. 1974;8:47-89.

6. ALMEIDA L. Alteraciones anatómico-funcionales en el Trastorno por déficit de la atención con hiperactividad. Salud Mental. 2005;28(3): 1 .

7. ESTÉVEZ-GONZÁLEZ A, GARCÍA-SÁNCHEZ C, JUNQUÉ C. La atención: una compleja función cerebral. Revista de neurología. 1997;25(148):1989-97.

8. SÁNCHEZ-CARPINTERO R, NARBONA J. Revisión conceptual del sistema ejecutivo y su estudio en el niño con trastorno por déficit de atención e hiperactividad. Revista de Neurología. $2001 ; 33(1): 47-53$.

9. GRANT DA, BERG E. A behavioral analysis of degree of reinforcement and ease of shifting to new responses in a Weigl-type card-sorting problem. Journal of experimental psychology. 1948;38(4):404.

10. STROOP JR. Studies of interference in serial verbal reactions. Journal of experimental psychology. 1935; 18(6):643.

11. GOLDEN CJ. A group version of the Stroop Color and Word Test. Journal of personality assessment. 1975;39(4):386-8.

12. LI F, HE N, LI Y, CHEN L, HUANG X, LUI S, ET AL. Intrinsic brain abnormalities in attention deficit hyperactivity disorder: a resting-state functional MR imaging study. Radiology. 2014;272(2):514-23.

13. DELGADO-MEJÍA I, ETCHEPAREBORDA MC. Trastornos de las funciones ejecutivas. Diagnóstico y tratamiento. Revista de neurología. 2013;57(1):95-103.

14. ABAD-MAS L, RUIZ-ANDRÉS R, MORENO-MADRID F, HERRERO R, SUAY E. Intervención psicopedagógica en el trastorno por déficit de atención/hiperactividad. Revista de Neurología. 2013;57(Supl 1):S193-203. 